\title{
Increasing role of imaging in differentiating MS from non-MS and defining indeterminate borderline cases
}

\author{
Maciej Juryńczyk ${ }^{1}$, Paweł Jakuszyk ${ }^{1}$, Iwona Kurkowska-Jastrzębska², Jacqueline Palace \\ ${ }^{1}$ Laboratory of Brain Imaging, Nencki Institute of Experimental Biology, Polish Academy of Sciences, Warsaw, Poland \\ ${ }^{2} 2^{\text {nd }}$ Department of Neurology, Institute of Psychiatry and Neurology, Warsaw, Poland \\ ${ }^{3}$ Nuffield Department of Clinical Neurosciences, University of Oxford, United Kingdom
}

\begin{abstract}
Multiple sclerosis (MS) is a heterogenous condition with differences between patients regarding disease presentation, imaging features, disease activity, prognosis and treatment responses. Following the discovery of new biomarkers, the concept of MS has evolved, with syndromes previously considered to be its variants now recognised as separate entities, including aquaporin-4 (AQP4)-antibody (Ab) neuromyelits optica spectrum disorders (NMOSD), and myelin oligodendrocyte glycoprotein (MOG)-Ab disease (MOGAD). In line with their distinct pathology, the newly emerging conditions have imaging characteristics which are dissimilar to typical MS. Progress in reclassifying such demyelinating CNS conditions has highlighted the challenge in meaningful categorisation of atypical presentations at the borders of $\mathrm{MS}$, such as antibody-negative neuromyelitis optica-like syndromes, tumefactive demyelinating lesions, or Balo's concentric sclerosis.

In this review, we discuss the increasing role of imaging in distinguishing MS from non-MS CNS inflammatory/demyelinating conditions and defining undetermined borderline cases. This progress relies both on better characterisation of imaging features of these conditions on conventional imaging in terms of their appearance and location, as well as on the implementation of novel image acquisition and/or post-processing techniques allowing for more in-depth lesion assessment, including the presence of a central vein sign or paramagnetic rim.
\end{abstract}

Key words: multiple sclerosis, neuromyelits optica

(Neurol Neurochir Pol 2022; 56 (3): 210-219)

\section{Introduction}

Multiple sclerosis (MS) is a chronic demyelinating inflammatory disease of the central nervous system (CNS) primarily driven by autoimmune response of $\mathrm{T}$ cells directed against myelin antigens [1]. More recently, the role of $B$ cells has been emphasised as evidenced by the presence of oligoclonal bands in the CSF and the beneficial effect of B cell-depleting therapies, including ocrelizumab, on disease activity [1].

There is no single diagnostic biomarker in MS, and the diagnosis relies on finding the evidence of disease dissemination, in the absence of a better explanation, which is encapsulated in the revised diagnostic criteria [2]. Brain magnetic resonance imaging (MRI) plays a major role in the diagnosis as it can show both dissemination in time - when contrast-enhancing lesions co-exist with non-contrast-enhancing lesions on the same scan or when new lesions emerge on a follow-up scan - and in space - when the scan shows multiple lesions in different regions of the brain and/or spinal cord. The presence of unmatched oligoclonal bands in the CSF suggestive of intrathecal antibody production also indicates dissemination of the disease process in time [2].

This classical approach to the diagnosis of MS has its limitations, because conditions recently recognised as separate from MS such as AQP4-antibody (AQP4- $\mathrm{Ab}$ ) neuromyelits optica spectrum disorders (NMOSD) and myelin

Address for correspondence: Maciej Juryńczyk, Laboratory of Brain Imaging, Nencki Institute of Experimental Biology, Polish Academy of Sciences, Warsaw, Poland; e-mail: m.jurynczyk@nencki.edu.pl 
oligodendrocyte glycoprotein (MOG)-Ab disease (MOGAD) may have overlapping clinical and radiological presentation and disease course with that of MS and may fulfill the diagnostic criteria for MS. All three conditions typically present with relapses of optic neuritis, transverse myelitis and brain/ brainstem attacks which are associated with inflammatory lesions on brain and spinal cord imaging $[3,4]$.

Serum testing for antibodies against AQP4 and MOG is the gold standard for the diagnosis of NMOSD and of MOGAD, respectively [5,6]. Antibody assays are, however, not widely available, and results usually take a long time to arrive and might be inconclusive or inaccurate, in particular if fixed cell-based assays or ELISA are used [7, 8]. CSF testing can also be helpful but has its limitations: oligoclonal bands unmatched for serum are a hallmark of MS but can be found in up to $30 \%$ of NMOSD and MOGAD patients $[9,10]$.

Taking all these caveats into account, imaging is often the main source of information for disease diagnosis. Not only are the imaging features crucial for the decision as to whether to test for antibodies, but also to the interpretation of the results. If assays are not available, or come back negative or positive using less accurate assays, then imaging becomes the main diagnostic tool.

\section{Longitudinally extensive transverse myelitis (LETM) is strong argument against diagnosis of MS}

Acute severe transverse myelitis alongside bilateral optic neuritis has been recognised as the classical clinical feature of neuromyelitis optica since its first description by Devic [9]. The clinical severity of transverse myelitis in NMO matches longitudinal extension of spinal cord lesions (termed LETM) which span at least three segments of the spinal cord and are associated with cord swelling and hypointensity in the central part of the cord seen on T1-weighted images [11]. LETM has been found to be such a common (91\%) and characteristic feature of NMO that it was incorporated into its first criteria, published before AQP4 antibody's discovery in 1999 [11]. LETM sets NMO apart from MS where transverse myelitis is accompanied by short-segment, lateral lesions in the spinal cord. LETM is extremely rare in MS but can be mimicked by LETM-like appearance of the coalescing short segment lesions [12]. To avoid misinterpretation, careful scrutiny of the scan should include both sagittal and axial images. It should be noted that while LETM is the predominant form of myelitis in both AQP4-Ab NMOSD and MOGAD, short-segment myelitis can be seen in both conditions, and does not exclude the consideration of antibody testing $[13,14]$.

LETM occurs both in AQP4-Ab NMOSD and MOGAD, and despite some differences none of the LETM features appear to be exclusive for any of the two [14-16]. Importantly, similar to AQP4-Ab NMOSD, LETM lesion in MOGAD can extend into the brainstem and involve the dorsal medulla. Conus involvement occurs in around $40 \%$ of patients and is more frequent than in AQP4-Ab NMOSD (c.12\%) [14, 17]. The most important clue might come from brain imaging showing acute disseminated encephalomyelitis (ADEM)-like lesions, typical of MOGAD and seen in up to $44 \%$ of patients with MOG-Ab associated myelitis [14, 18]. Contrast enhancement is frequent in AQP4-Ab LETM lesions and can take the form of ring-enhancement [19]. Contrast enhancement has also been reported in MOG-Ab LETM, but with varied frequency between studies $[14,17]$.

LETM lesions are also seen in monophasic and relapsing idiopathic antibody-negative transverse myelitis and in double antibody negative NMOSD. In a retrospective study including a small number of seronegative NMO patients $(n=5)$, seronegative LETM had similar features to AQP4-Ab-associated LETM [15]. In a recent study classifying double antibody-negative MS/NMOSD overlap patients in an unsupervised way based on clinical and MRI measures, 2 / 4 identified subgroups included patients with a previous history of LETM [20]. One of these subgroups included 'classic NMO-like' patients with bilateral optic neuritis and no evidence of brain damage. The other group is known as 'NMO-like with brain involvement' as patients had significant cerebral white and grey matter damage and a history of NMO-like brain lesions. This dichotomy within antibody-negative LETM patients suggests that double-negative NMOSD might be heterogenous and contain distinct pathological subgroups [20]. Importantly, long spinal cord lesions are also seen outside of NMOSD [21]. For example, in spinal cord sarcoidosis they are characterised by dorsal cord subpial enhancement extending over at least two vertebral segments and persistent enhancement present for 2+ months [22]. If dorsal-subpial enhancement co-occurs with central canal enhancement, the lesion often resembles a trident head on axial sequences. This has been termed the 'trident sign' [23].

\section{Importance of defining appearance of imaging lesions for diagnostic purposes}

Early attempts at incorporating imaging features into the MS diagnostic criteria focused on predicting the development of MS in those with clinically isolated syndrome, rather than on excluding its mimics [24, 25]. Paty's criteria included at least four lesions (of any type) or at least three if one of them was periventricular [25]. These criteria were highly sensitive but lacked specificity. Barkhof et al. [26] studied in more detail cut-off points for the number of particular types of lesions needed to fulfill the criteria, and proposed highly sensitive and specific criteria.

One of the challenges when establishing any set of criteria is the selection of features. For example, periventricular lesions which are ovoid, perpendicular to the ventricles, and extend into the adjacent white matter have long been considered highly typical of MS (and termed 'Dawson fingers' as reflecting 
the perivenular distribution of MS lesions on histopathology first described by Dawson), but were rarely studied as a separate diagnostic criterion [27]. Matthews et al. [28] proposed a more objective and less arbitrary approach to criteria selection when they compared brain lesion probability maps in $50 \mathrm{MS}$ and $44 \mathrm{AQP} 4-\mathrm{Ab}$ NMOSD patients. This comparison of distribution and morphology of lesions led to the identification of one out of three criteria in favour of MS diagnosis vs. NMOSD, consisting of: at least one lesion adjacent to the body of the lateral ventricle and in the inferior temporal lobe; or the presence of a curved juxtacortical lesion; or a Dawson finger-type lesion [28]. These criteria could distinguish MS from AQP4-Ab NMOSD with very high sensitivity of $92 \%$ and very high specificity of $96 \%$ in the original cohort. A further study validated their performance in an independent multicentre European cohort and, importantly, showed their high specificity in distinguishing MS from MOGAD [29]. The criteria, sometimes also incorporating longitudinally extensive transverse myelitis (LETM) on spinal cord imaging, have been further validated against NMOSD and MOGAD in Asian and Latin American cohorts [30-32].

\section{Fluffy infratentorial lesions and other lesions typical of MOGAD}

Following the objective brain lesion mapping comparison of MS and AQP4-Ab NMOSD [28], a later study also included MOGAD patients to study relationships between the three conditions [33]. This time an alternative approach was used, where patients were scored on a set of 29 pre-selected features to build models discriminating between the three conditions and identify the best discriminators [33]. MS clearly differed from both NMOSD and MOGAD as evidenced by predictive discriminant models. Interestingly, AQP4-Ab vs. MOGAD model was non-predictive, which pointed to overlapping brain appearances of antibody-mediated conditions despite their different cellular targets. Fluffy infratentorial lesions were the most promising discriminator, and were present in $57 \%$ of MOGAD compared to $27 \%$ of AQP4-Ab NMOSD patients. These lesions were rare in MS (12\%). Fluffy infratentorial lesions in MOGAD patients were more often located in cerebellar peduncles ( $26 \% v s .9 \% \mathrm{AQP} 4-\mathrm{Ab}$ NMOSD). A recent study also reported that a cerebellar peduncle lesion increases the likelihood of MOGAD vs. NMOSD [34]. Other brain imaging lesions which have been reported to be more frequent in MOGAD vs. AQP4-Ab NMOSD include cortical grey/juxtacortical white matter lesions [35].

Brain lesions are an important aspect of childhood MOG$\mathrm{AD}$, where the disease often presents as ADEM [10]. Lesions are large, poorly demarcated and bilateral, typically affecting white and grey matter including thalamus and basal ganglia, but can also be seen in the brainstem and spinal cord. Unlike MS, periventricular lesions are typically absent. The appearances are similar to those seen in MOG-Ab-negative ADEM.
In one study including a total of 33 children, patients with MOG-Ab-associated ADEM had the involvement of more anatomical areas including the spinal cord characterised by LETM, and more often had a complete resolution of lesions compared to MOG-Ab-negative cases [36].

A different type of brain involvement has been described in a unique presentation of MOGAD characterised by unilateral cortical encephalitis with seizures [37]. Lesions are associated with mild swelling and are limited to the cortex. They are best seen on FLAIR-weighted images, but might partially enhance on T1-weighted imaging after contrast [37]. Of interest, seizures have also been described in MOGAD in patients with normal brain MRI [38]. Similarly, on rare occasions (c. 10\%) transverse myelitis can be MRI-negative in MOGAD, in particular in the early stages of acute myelitis [39].

\section{AQP4-Ab NMOSD-associated brain lesions}

Brain lesions associated with AQP4-Ab NMOSD have been well characterised and include diencephalic lesions adjacent to the $3^{\text {rd }}$ ventricle, dorsal medulla lesions (including area postrema), peripendymal lesions surrounding the lateral ventricles, extensive hemispheric lesions, and long corticospinal tract lesions [40]. Such lesions are extremely rare in MS, and where they occur argue strongly against a diagnosis of MS. It has been proposed that the location and configuration of NMOSD lesions could be explained in the main by high expression of AQP4 in the affected regions. Area postrema is a good example, as it has a predilection for imaging lesions and is known to be rich in AQP4 [41]. However, as NMOSD-like lesions might occur in MOGAD [33, 42], alternative explanations for their formation are possible, e.g. linked with the point of entry of antibodies to the CNS. It is a matter of debate whether AQP4-Ab NMOSD has any unique imaging features which never occur in MOGAD.

\section{Optic nerve imaging}

While brain imaging appearances do not unequivocally discriminate between AQP4-Ab NMOSD and MOGAD, optic nerve features might be more distinctive in those who present with optic neuritis. In particular, perineural enhancement defined as contrast enhancement of the optic nerve sheath (sometimes also of the surrounding soft tissue) has been reported to be a specific feature of MOG-Ab-associated optic neuritis occurring in around half of patients and may be the cause of MOGAD optic neuritis-associated headache [43-45]. Both AQP4-Ab NMOSD and MOGAD are characterised by longitudinally extensive (involving more than $50 \%$ of the length of the nerve) optic nerve lesions. In MOGAD, long lesions have a tendency to affect the anterior segment of the nerve and co-occur with optic nerve swelling on fundoscopy, while AQP4-Ab optic neuritis has a predilection for posterior segments and the optic chiasm [40,44]. Optic chiasm lesions 
also occur in MOGAD where they are usually a part of the longitudinally extensive involvement of the whole nerve starting in the orbit [46]. Longitudinally extensive lesions of the optic nerve are rare in MS $[47,48]$. It should also be noted that both NMOSD and MOGAD have a tendency for bilateral optic nerve involvement, which would be very unusual in MS [3].

\section{Atypical demyelination with an unclear link to MS}

Recognition of AQP4-Ab NMOSD and MOGAD as new disease entities having a separate non-MS-like radiological appearances has sparked interest in other types of atypical demyelination with an unclear link to MS, in particular tumefactive demyelinating lesions (TDLs) and Balo's concentric sclerosis $[49,50]$.

TDLs are radiologically defined as lesions greater than $2 \mathrm{~cm}$ in diameter and are typically seen on T1- and T2-weighted imaging (as hypointense and hyperintense, respectively) [51, 52]. They are usually associated with minimal surrounding oedema and a T2-hypointense rim (Fig. 1D). Contrast enhancement is seen in most cases and can be ring-like (closed or open ring), homogenous or heterogenous/punctiform. TDLs at onset presentation can occur in isolation or in the context of MS, NMOSD, MOGAD or ADEM (Fig. 2). The occurrence of TDLs in MS does not seem to indicate a highly active relapsing course of the disease or predict a poor long-term prognosis [53]. In a recent study including 75 patients with pathologically confirmed MS following a brain biopsy (in 62 the biopsied lesion size was at least $2 \mathrm{~cm}$ ), long-term clinical and imaging outcomes were comparable to those with typical MS [54]. When TDLs occur in isolation, differential diagnosis is challenging and includes abcesses, tumours and progressive multifocal leukoencephalopathy. Serial imaging might be of help as diffusion-weighted imaging can show evolving restricted diffusion at the lesion edge (Fig. 1E). Otherwise, workup might include brain biopsy. In a retrospective study including 16 patients with isolated tumefactive lesion at onset after a median follow-up of more than 5 years, 10 patients remained monophasic at final follow-up, one had recurrence of TDL, and five received the diagnosis of MS [55]. AQP4- and MOG-Abs have been reported in patients with isolated TDL and both antibodies should be tested in such cases [56,57]. If patients with relapsing TDL are double antibody-negative and do not fulfill the MS criteria, the classification and treatment are unclear but most clinicians would probably opt for B cell-depleting therapies.

TDLs can sometimes present as Balo's concentric sclerosis. Typical features include alternating and concentric bands within white matter lesions identified on either T1-, T2-, or diffusion-weighted MRI and showing gadolinium enhancement. Alternating bands are characterised by at least two T2 hyperintense bands separated by one hypointense band. The number of $\mathrm{T} 2$ hyperintense bands can increase on subsequent

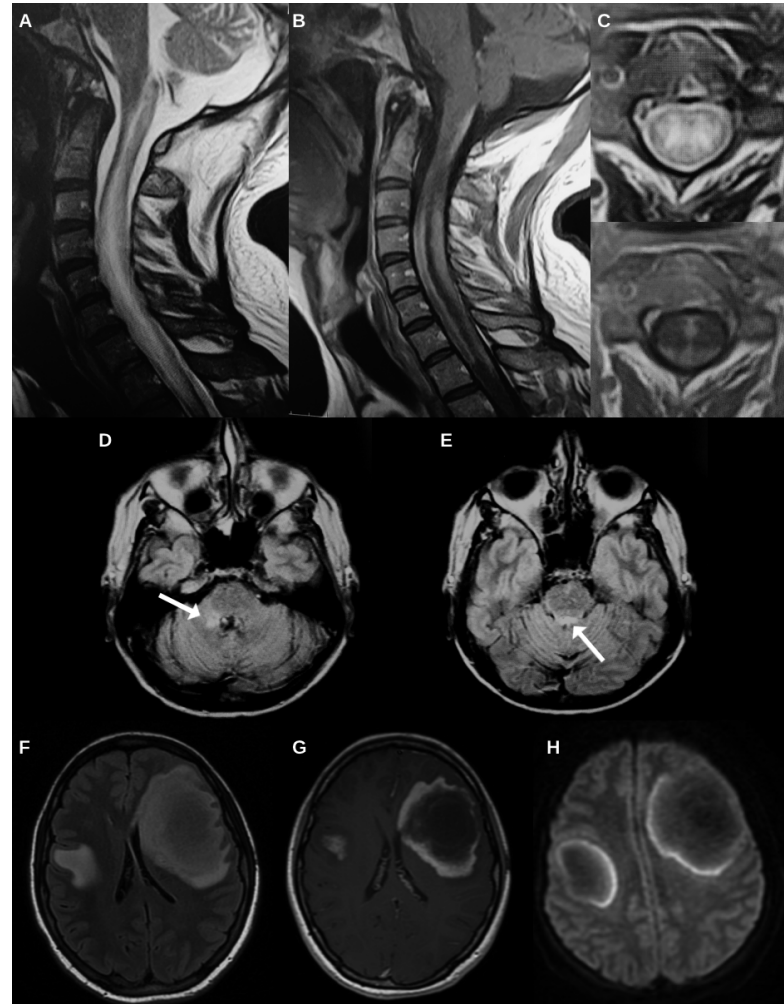

Figure 1.A-C. A 43-year old male had two attacks of LETM four months apart and subsequently developed progressive motor disability; A. T2-weighted sagittal image performed in acute phase shows cervical LETM extending to dorsal medulla; $B$. Lesion has accompanying contrast enhancement on post-gadolinium T1-weighted image; $\mathbf{C}$. On axial images, $\mathrm{T} 2$ hyperintensity is mainly restricted to grey matter (upper image) but gadolinium enhancement is more pronounced in anterior and posterior parts of cord. Patient repeatedly tested negative for both anti-AQP4 and anti-MOG serum antibodies; D-E. A 64-year old female with history of right optic neuritis presented with right-sided trigeminal neuralgia associated with fluffy infratentorial lesions in brainstem and right middle cerebral peduncle (arrows). Although presentation and imaging were highly suggestive of MOGAD, patient tested negative for MOG-Ab; F-H. An adult female presented with acute onset of left hemiparesis and speech disturbances; F. FLAIR imaging showed two large tumefactive lesions surrounded by mild oedema located in hemispheric white matter. Lesions showed open ring enhancement on post-contrast images $(\mathbf{G})$ and restricted diffusion at lesion edge $(\mathbf{H})$. A two-segment cervical spinal cord lesion was also present (not shown). Patient tested negative for both AQP4- and MOG-Abs, and following brain biopsy was diagnosed with ADEM (clinical case courtesy of Dr Beata Błażejewska-Hyżorek, $2^{\text {nd }}$ Department of Neurology, Institute of Psychiatry and Neurology, Warsaw, Poland). Abs - antibodies; AQP4 - aquaporin-4; LETM - longitudinally extensive transverse myelitis; MOG - myelin oligodendrocyte glycoprotein; MOGAD -MOG-Ab disease

scans performed within one month [50]. Evolution of signal abnormalities at the lesion edge is particularly interesting with diffusion restriction seen before contrast enhancement, which in turn is followed by the rim of T2 hyperintensity [58]. 


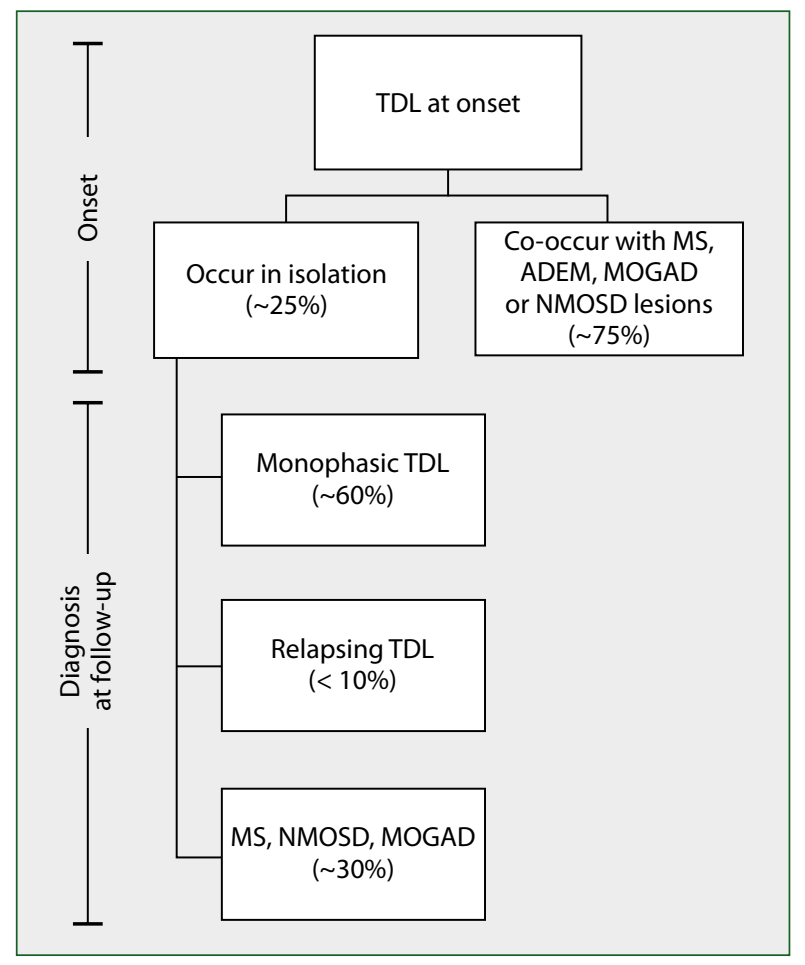

Figure 2. Possible clinical scenarios in patients with TDL on imaging at disease onset. ADEM - acute-disseminated encephalomyelitis; MOGAD - myelin oligodendrocyte glycoprotein antibody disease; MS - multiple sclerosis; NMOSD - neuromyelitis optica spectrum disorders; TDL - tumefactive demyelinating lesions

A Balo's concentric sclerosis lesion can be isolated and monophasic but usually occurs in conjunction with MS-like lesions or precedes the occurrence of more typical MS-like lesions. Diagnosis is thus clearer than in TDLs due to the characteristic MRI appearance and correct interpretation preventing unnecessary brain biopsy in solitary lesions.

\section{New silent lesions outside of relapse}

In both AQP4-Ab NMOSD and MOGAD, silent brain lesions during acute attacks are well recognised. For example, in an early study, $44 \%$ of patients with MOGAD who presented with neuromyelitis optica phenotype (simultaneous optic neuritis and transverse myelitis) were found to have silent ADEM-like lesions on brain MRI [18]. Silent lesions occurring outside of relapse are however unusual in antibody-mediated conditions. This holds true for both spinal cord and brain imaging and is very different from MS, where silent asymptomatic lesions on follow-up scans are a typical finding and reflect ongoing disease activity. In five different studies including a total of 82 MOGAD patients scanned at different follow-up points, not a single silent spinal cord lesion was observed in any of the patients [17, $18,35,59,60]$. In a paediatric study including a prospective incident cohort of 74 MOG-Ab children with serial brain MRI scans over a median of five years from presentation, silent brain lesions were identified infrequently (14\% of patients) and most commonly within the first months after presentation [61].

In line with these observations, and in contrast to MS, serial routine MRI in antibody-mediated conditions is generally not considered useful for disease activity monitoring [62].

\section{Progress in structural imaging helps differential diagnosis in neuroimmunology clinics}

Recent progress in structural imaging incorporating new pulse sequences and post-processing techniques provides deeper understanding of the underlying tissue damage. This allows better characterisation of various aspects of MS lesions such as subpial or intracortical demyelination, lesion formation around the veins, and chronic activity at the lesion edge.

\section{Cortical lesions}

Cortical lesions represent foci of cortical demyelination and are a characteristic pathological feature of MS [63]. They can be small and purely intracortical, involve both grey and white matter or extend within the cortex from the surface downwards [64]. Cortical lesions are clinically relevant as they correlate better with cognitive impairment in MS than the more typical MRI white matter lesions [65]. They also have predictive value for disability progression [66].

Reliable detection of cortical lesions is challenging in clinical practice as cortical lesions are difficult to visualise using routine T2-weighted or FLAIR imaging. This problem can be partially overcome by the use of a double inversion recovery (DIR) sequence which improves the contrast between the lesion and the normal cortex and suppresses the signal both from the white matter and the CSF [67]. Phase-sensitive inversion recovery (PSIR) is another widely used technique for cortical lesion detection (Fig. 3A), allowing for good resolution in a shorter acquisition time than DIR [68]. These optimised sequences are able to detect up to $25 \%$ of cortical lesions compared to the golden standard of histopathology [69]. Although this percentage might seem low, it is expected to correlate well with the total number of cortical lesions in an individual patient [69].

Cortical lesions occur in MS, but are rare in AQP4-Ab NMOSD $[16,70]$. In a recent comparative study, cortical/ /juxtacortical lesions were seen in $65 \%$ of MS patients and in $16 \%$ of MOGAD patients, but were absent in AQP4-Ab NMOSD [16]. Interestingly, all cortical lesions in MOGAD were of the mixed cortical/juxtacortical type, while MS patients had both leukocortical and purely cortical lesions [16]. Others have also reported cortical/juxtacortical lesions in a minority of MOGAD patients [35, 42]. Pathology studies have also demonstrated the presence of both intracortical, leukocortical and subpial demyelinating lesions in MOGAD, in particular in those with ADEM and cortical encephalitis [71, 72]. It should 
be noted that MS-like small and localised cortical lesions are different on MRI from oedematous cortical lesions reported on FLAIR images in MOG-Ab-associated unilateral cortical encephalitis [37]. In a recent prospective study including 25 patients with overlapping features of NMOSD and MS, no cortical lesions were identified using DIR in those who received the clinical diagnosis of antibody-negative NMOSD in a highly specialised NMO clinic [20].

\section{Leptomeningeal enhancement (LME)}

LME is a recently described imaging sign that has been proposed to reflect the presence of $\mathrm{B}$ cell follicles in the meninges of MS patients $[73,74]$. LME is predominantly identified in patients with progressive forms of MS. Since B cell follicles have been implicated in the formation of cortical demyelination, LME is expected to co-occur with cortical lesions on imaging. This link however has not been completely proven, as only a subset of studies has shown a higher frequency of cortical lesions in those with $\operatorname{LME}[75,76]$. It is also worth noting that LME has been detected not only in MS patients but also in other conditions, for example those of infectious or vascular origin. Importantly, LME was retrospectively demonstrated on post-contrast T1-weighted and FLAIR images as a sign of leptomeningeal blood-brain barrier disruption in 11 AQP4-Ab NMOSD patients on clinical scans performed at onset attack or relapse [77]. Such discrepancies call for caution in the interpretation of LME.

\section{Central vein sign (CVS)}

The presence of a vein in the centre of a demyelinating lesion is a pathological hallmark of MS. In recent years, this has been able to be visualised using imaging and used to differentiate MS from its mimics (Fig. 3B).

The visualisation of a CVS is typically performed by combining two images acquired in a different way: one that depends on the paramagnetic properties of haemoglobin in the vessels (T2*-weighted or susceptibility-weighted imaging), and one that removes signal from the CSF and offers good contrast from the lesions (FLAIR) [78]. Gadolinium injection is sometimes used to enhance the visibility of vessels [79]. Around $70-80 \%$ of MS lesions are CVS-positive and this percentage increases with the proximity of the lesion to the lateral ventricles [78]. Lesions with CVS have been identified in non-MS inflammatory conditions of the CNS, including AQP4-Ab NMOSD [80], MOGAD [81], Susac syndrome [82] or CNS inflammatory vasculopathies [79], but represent a minority of lesions. Interestingly, CVS was not detected in any of nine patients with systemic lupus erythematosus and brain involvement [79]. It is worth noting that some lesions in MS mimics might contain a vein, but its location is not central as reported in AQP4-Ab NMOSD [80]. The presence of CVS in MS mimics has led to the introduction of cut-off

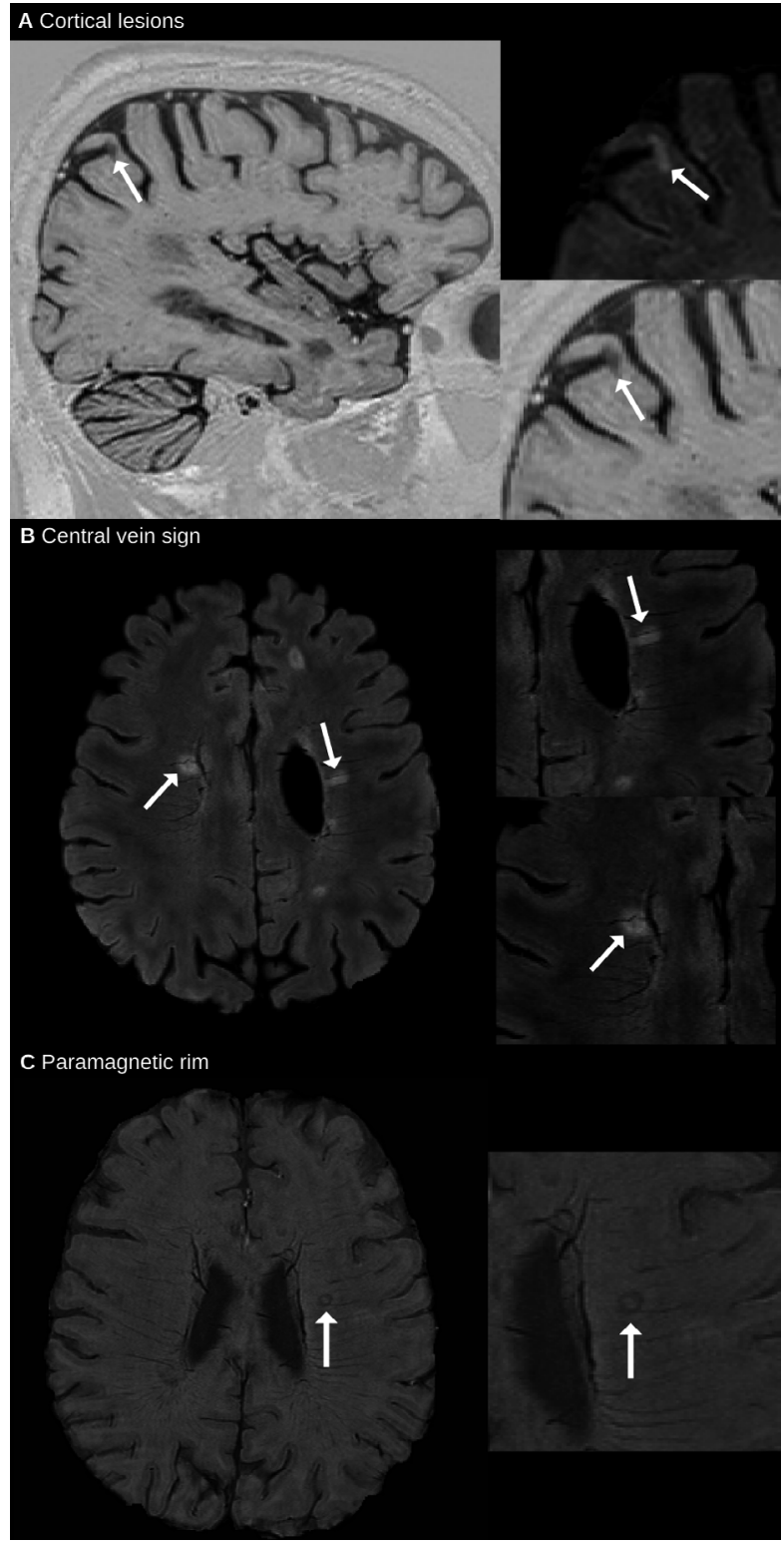

Figure 3. Non-conventional imaging findings in patients with MS and radiologically isolated syndrome; $\mathbf{A}$. A cortical lesion seen on phase-sensitive inversion recovery sequence (marked with arrow on left, enlarged on right lower image) in a male entering secondary progressive MS. Same lesion is also identified on FLAIR (right upper image); B. Two MS-typical ovoid, well-demarcated lesions perpendicular to lateral ventricles and centred by veins as shown on FLAIR* images (marked with arrows and enlarged on right) in an adult female with radiologically isolated syndrome; C. Lesion with hypointense paramagnetic rim on high-resolution susceptibility weighted image (marked with arrow and enlarged on right) in adult male with MS. MS - multiple sclerosis

rules for CVS positivity including a ' $40 \%$ threshold' (i.e. at least $40 \%$ of lesions positive for CVS) [83] and a 'six lesions rule' (where at least six lesions have veins in the centre) [84]. Apart from the diagnosis, CVS can also be potentially useful in predicting the occurrence of CIS and ultimate MS diagnosis 
in those with incidental MS-like white matter lesions (known as radiologically isolated syndrome).

\section{Paramagnetic rim sign (PRS)}

While central veins can be identified in both acute and chronic lesions, the presence of a paramagnetic rim is associated with chronic active lesions and corresponds to iron-rich, activated microglia and macrophages at the lesion edge [85]. PRS is therefore a sign of ongoing inflammation occurring behind the intact blood-brain barrier, as opposed to acute inflammation seen on T1-weighted post-contrast images. The detection of PRS requires information from phase images, which show local signal distortion at the lesion edge caused by paramagnetic iron. It is seen as hypointense on SWI and hyperintense on quantitative susceptibility imaging. Interestingly, PRS lesions are stable or expand slowly over time [86]. Their presence is correlated with worse motor and cognitive disability outcomes and higher levels of serum neurofilament light chain, a marker of axonal damage $[86,87]$. This correlation is particularly striking if there are four or more lesions with a rim [86, 87]. Recently, an alternative method of detecting rims has been proposed which involves the use of a double inversion recovery sequence suppressing both CSF and grey matter signal [88]. Dark rims detected by this technique showed $97 \%$ specificity in discriminating RRMS from non-MS white matter lesions when at least two rim lesions were present [88].

Reflecting ongoing inflammation, PRS is an excellent candidate for a biomarker to predict disease course and monitor treatment efficacy, but can also be useful diagnostically (Fig. 3C). In a recent international multicentre study including 438 individuals with various neurological conditions, paramagnetic rim lesions were present in 52\% of MS patients compared to 7\% of patients with a non-MS diagnosis, which gave this feature a very high specificity of $93 \%$ in differentiating MS from nonMS [89]. In particular, rims were very rarely (less than 5\%) reported in NMOSD lesions [80, 90]. Similar to CVS, PRS can be detected in patients with radiologically isolated syndrome, pointing to the existence of chronic active inflammation in this asymptomatic group [91]. Their significance in predicting the development of clinical disease is however currently unknown, and requires further studies.

\section{Conclusions and future prospects}

The differential diagnosis of MS has recently broadened to include the newly recognised conditions AQP4-antibody NMOSD and MOGAD. More attention has also been paid to double antibody-negative patients with overlapping features of MS and NMOSD as being diagnostically and therapeutically challenging [92].

Given the occurrence of borderline, undetermined and atypical cases, a relative lack of biomarkers, limited access to optimised antibody tests, and the likelihood of false positive and false negative results, the role of imaging in differential diagnosis is critical. A compelling need for precise imaging has been paralleled by the characterisation of new MS imaging signs requiring novel types of image acquisition or image post-processing methods, which can be applied reliably on 3T scanners. These features have either high specificity for MS (such as paramagnetic rim or cortical lesions) or need to be determined by a cut-off level (central vein sign). The identification is currently largely visual, but initial attempts at automatic detection have been made [93, 94]. Limitations still exist; for example, paramagnetic rim and CVS are best assessed in well-demarcated lesions whereas larger, confluent and merging lesions are more challenging. Detection of cortical lesions (in particular of the intracortical and subpial types) is still suboptimal even with optimised inversion recovery sequences.

Future progress will focus on increasing detection sensitivity, unifying acquisition and analysis protocols, and identifying new imaging signs and their automated detection.

Acknowledgements: MaciejJuryńczyk and Paweł Jakuszyk receive funding from the Polish National Agency For Academic Exchange ('Polish Returns' programme, PPN/PPO/2020/1/00043/U/00001) and the National Science Centre (providing a research component of the programme) 2021/01/1/NZ5/00008.

\section{References}

1. Thompson A, Baranzini S, Geurts J, et al. Multiple sclerosis. Lancet. 2018; 391(10130): 1622-1636, doi: 10.1016/s01406736(18)30481-1, indexed in Pubmed: 29576504.

2. Thompson A, Banwell B, Barkhof $F$, et al. Diagnosis of multiple sclerosis: 2017 revisions of the McDonald criteria. Lancet Neurol. 2018; 17(2): 162-173, doi: 10.1016/s1474-4422(17)30470-2, indexed in Pubmed: 29275977.

3. Juryńczyk M, Craner M, Palace J. Overlapping CNS inflammatory diseases: differentiating features of NMO and MS. J Neurol Neurosurg Psychiatry. 2015; 86(1): 20-25, doi: 10.1136/jnnp-2014-308984, indexed in Pubmed: 25248365.

4. Juryńczyk M, Jacob A, Fujihara K, et al. Myelin oligodendrocyte glycoprotein (MOG) antibody-associated disease: practical considerations. Pract Neurol. 2019; 19(3): 187-195, doi: 10.1136/practneurol-2017-001787, indexed in Pubmed: 30530724.

5. Wingerchuk DM, Banwell B, Bennett JL, et al. International Panel for NMO Diagnosis. International consensus diagnostic criteria for neuromyelitis optica spectrum disorders. Neurology. 2015; 85(2): 177-189, doi: 10.1212/WNL.0000000000001729, indexed in Pubmed: 26092914.

6. Waters P, Woodhall M, O'Connor KC, et al. MOG cell-based assay detects non-MS patients with inflammatory neurologic disease. Neurol Neuroimmunol Neuroinflamm. 2015; 2(3): e89, doi: 10.1212/ NXI.0000000000000089, indexed in Pubmed: 25821844.

7. Waters PJ, McKeon A, Leite MI, et al. Serologic diagnosis of NMO: a multicenter comparison of aquaporin-4-IgG assays. Neurology. 2012; 78(9): 665-671, doi: 10.1212/WNL.0b013e318248dec1, indexed in Pubmed: 22302543.

8. Reindl M, Schanda K, Woodhall M, et al. International multicenter examination of MOG antibody assays. Neurol Neuroimmunol neuroinflam- 
mation. Neurol Neuroimmunol Neuroinflamm. 2020; 7(2): e674, doi: 10.1212/NXI.0000000000000674, indexed in Pubmed: 32024795.

9. Wingerchuk DM, Lennon VA, Lucchinetti CF, et al. The spectrum of neuromyelitis optica. Lancet Neurol. 2007; 6(9): 805-815, doi: 10.1016/S1474-4422(07)70216-8, indexed in Pubmed: 17706564.

10. Jurynczyk M, Messina S, Woodhall MR, et al. Clinical presentation and prognosis in MOG-antibody disease: a UK study. Brain. 2017; 140(12): 3128-3138, doi: 10.1093/brain/awx276, indexed in Pubmed: 29136091.

11. Wingerchuk DM, Hogancamp WF, O'Brien PC, et al. The clinical course of neuromyelitis optica (Devic's syndrome). Neurology. 1999; 53(5): 1107-1114, doi: 10.1212/wnl.53.5.1107, indexed in Pubmed: 10496275.

12. Asnafi S, Morris PP, Sechi E, et al. The frequency of longitudinally extensive transverse myelitis in MS: a population-based study. Mult Scler Relat Disord. 2020; 37: 101487, doi: 10.1016/j.msard.2019.101487, indexed in Pubmed: 31707235.

13. Flanagan EP, Weinshenker BG, Krecke KN, et al. Short myelitis lesions in aquaporin-4-IgG-positive neuromyelitis optica spectrum disorders. JAMA Neurol. 2015; 72(1): 81-87, doi: 10.1001/jamaneurol.2014.2137, indexed in Pubmed: 25384099.

14. Dubey D, Pittock SJ, Krecke KN, et al. Clinical, radiologic, and prognostic features of myelitis associated with myelin oligodendrocyte glycoprotein autoantibody. JAMA Neurol. 2019; 76(3): 301-309, doi: 10.1001/jamaneurol.2018.4053, indexed in Pubmed: 30575890.

15. Kitley J, Leite MI, Küker W, et al. Longitudinally extensive transverse myelitis with and without aquaporin 4 antibodies. JAMA Neurol. 2013; 70(11): 1375-1381, doi: 10.1001/jamaneurol.2013.3890, indexed in Pubmed: 23999580.

16. Messina S, Mariano R, Roca-Fernandez A, et al. Contrasting the brain imaging features of MOG-antibody disease, with AQP4-antibody NMOSD and multiple sclerosis. Mult Scler. 2021 [Epub ahead of print]: 13524585211018987, doi: 10.1177/13524585211018987, indexed in Pubmed: 34048323.

17. Mariano R, Messina S, Kumar K, et al. Comparison of clinical outcomes of transverse myelitis among adults with myelin oligodendrocyte glycoprotein antibody vs aquaporin-4 antibody disease. JAMA Netw Open. 2019; 2(10): e1912732, doi: 10.1001/jamanetworkopen.2019.12732, indexed in Pubmed: 31596489.

18. Kitley J, Waters $\mathrm{P}$, Woodhall M, et al. Neuromyelitis optica spectrum disorders with aquaporin-4 and myelin-oligodendrocyte glycoprotein antibodies: a comparative study. JAMA Neurol. 2014; 71(3): 276283, doi: 10.1001/jamaneurol.2013.5857, indexed in Pubmed: 24425068.

19. Zalewski NL, Morris PP, Weinshenker BG, et al. Ring-enhancing spinal cord lesions in neuromyelitis optica spectrum disorders. J Neurol Neurosurg Psychiatry. 2017; 88(3): 218-225, doi: 10.1136/jnnp2016-314738, indexed in Pubmed: 27913626.

20. Juryńczyk M, Klimiec-Moskal E, Kong $\mathrm{Y}$, et al. Elucidating distinct clinico-radiologic signatures in the borderland between neuromyelitis optica and multiple sclerosis. J Neurol. 2021 [Epub ahead of print], doi: 10.1007/s00415-021-10619-1, indexed in Pubmed: 34043042.

21. Trebst C, Raab P, Voss EV, et al. Longitudinal extensive transverse myelitis - it's not all neuromyelitis optica. Nat Rev Neurol. 2011; 7(12): 688-698, doi: 10.1038/nrneurol.2011.176, indexed in Pubmed: 22045269.

22. Flanagan EP, Kaufmann TJ, Krecke KN, et al. Discriminating long myelitis of neuromyelitis optica from sarcoidosis. Ann Neurol. 2016;
79(3): 437-447, doi: 10.1002/ana.24582, indexed in Pubmed: 26677112.

23. Zalewski NL, Krecke KN, Weinshenker BG, et al. Central canal enhancement and the trident sign in spinal cord sarcoidosis. Neurology. 2016; 87(7): 743-744, doi: 10.1212/WNL.0000000000002992, indexed in Pubmed: 27527540.

24. Poser CM, Paty DW, Scheinberg L, et al. New diagnostic criteria for multiple sclerosis: guidelines for research protocols. Ann Neurol. 1983; 13(3): 227-231, doi: 10.1002/ana.410130302, indexed in Pubmed: 6847134.

25. Paty DW, Oger JJ, Kastrukoff LF, et al. MRI in the diagnosis of MS: a prospective study with comparison of clinical evaluation, evoked potentials, oligoclonal banding, and CT. Neurology. 1988; 38(2): 180-185, doi: 10.1212/wnl.38.2.180, indexed in Pubmed: 3340277.

26. Barkhof F, Filippi M, Miller DH, et al. Comparison of MRI criteria at first presentation to predict conversion to clinically definite multiple sclerosis. Brain. 1997; 120 (Pt 11): 2059-2069, doi: 10.1093/brain/120.11.2059, indexed in Pubmed: 9397021.

27. Simon JH, Holtås SL, Schiffer RB, et al. Corpus callosum and subcallosal-periventricular lesions in multiple sclerosis: detection with MR. Radiology. 1986; 160(2): 363-367, doi: 10.1148/radiology.160.2.3726114, indexed in Pubmed: 3726114.

28. Kister I, Ge Y, Herbert J, et al. Distinction of seropositive NMO spectrum disorder and MS brain lesion distribution. Neurology. 2013; 81(22): 1966.

29. Juryńczyk M, Tackley G, Kong Y, et al. Brain lesion distribution criteria distinguish MS from AQP4-antibody NMOSD and MOG-antibody disease. J Neurol Neurosurg Psychiatry. 2017; 88(2): 132-136, doi: 10.1136/jnnp-2016-314005, indexed in Pubmed: 27951522.

30. Bensi C, Marrodan M, González A, et al. Brain and spinal cord lesion criteria distinguishes AQP4-positive neuromyelitis optica and MOG-positive disease from multiple sclerosis. Mult Scler Relat Disord. 2018; 25: 246-250, doi: 10.1016/j.msard.2018.08.008, indexed in Pubmed: 30144694.

31. Hyun JW, Huh SY, Shin HJ, et al. Evaluation of brain lesion distribution criteria at disease onset in differentiating MS from NMOSD and MOG-IgG-associated encephalomyelitis. Mult Scler. 2019; 25(4): 585-590, doi: 10.1177/1352458518761186, indexed in Pubmed: 29512413.

32. Carnero Contentti E, Marques VD, Soto de Castillo I, et al. Brain and spinal MRI features distinguishing MS from different AQP4 antibody serostatus NMOSD at disease onset in a cohort of Latin American patients. Mult Scler. 2020; 26(8): 945-954, doi: 10.1177/1352458519849517, indexed in Pubmed: 31124748.

33. Jurynczyk M, Geraldes R, Probert F, et al. Distinct brain imaging characteristics of autoantibody-mediated CNS conditions and multiple sclerosis. Brain. 2017; 140(3): 617-627, doi: 10.1093/brain/ aww350, indexed in Pubmed: 28364548.

34. Matsumoto Y, Misu T, Mugikura S, et al. Distinctive lesions of brain MRI between MOG-antibody-associated and AQP4-antibody-associated diseases. J Neurol Neurosurg Psychiatry. 2020 [Epub ahead of print], doi: 10.1136/jnnp-2020-324818, indexed in Pubmed: 33380491.

35. Salama S, Khan M, Shanechi A, et al. MRI differences between MOG antibody disease and AQP4 NMOSD. Mult Scler. 2020; 26(14): 18541865, doi: $10.1177 / 1352458519893093$, indexed in Pubmed: 31937191.

36. Baumann M, Sahin K, Lechner C, et al. Clinical and neuroradiological differences of paediatric acute disseminating encephalomyelitis with and without antibodies to the myelin oligodendrocyte glycoprotein. 
J Neurol Neurosurg Psychiatry. 2015; 86(3): 265-272, doi: 10.1136/ jnnp-2014-308346, indexed in Pubmed: 25121570.

37. Ogawa R, Nakashima I, Takahashi T, et al. MOG antibody-positive, benign, unilateral, cerebral cortical encephalitis with epilepsy. Neurol Neuroimmunol Neuroinflamm. 2017; 4(2): e322, doi: 10.1212/ NXI.0000000000000322, indexed in Pubmed: 28105459.

38. Ramanathan S, O'grady GL, Malone S, et al. Isolated seizures during the first episode of relapsing myelin oligodendrocyte glycoprotein antibody-associated demyelination in children. Dev Med Child Neurol. 2019; 61(5): 610-614, doi: 10.1111/dmcn.14032, indexed in Pubmed: 30221764.

39. Sechi E, Krecke KN, Pittock SJ, et al. Frequency and characteristics of MRI-negative myelitis associated with MOG autoantibodies. Mult Scler. 2021; 27(2): 303-308, doi: 10.1177/1352458520907900, indexed in Pubmed: 32103708.

40. Kim HJ, Paul F, Lana-Peixoto MA, et al. Guthy-Jackson Charitable Foundation NMO International Clinical Consortium \& Biorepository. MRI characteristics of neuromyelitis optica spectrum disorder: an international update. Neurology. 2015; 84(11): 1165-1173, doi: 10.1212/ WNL.0000000000001367, indexed in Pubmed: 25695963.

41. Popescu BF, Lennon VA, Parisi JE, et al. Neuromyelitis optica unique area postrema lesions: nausea, vomiting, and pathogenic implications. Neurology. 2011; 76(14): 1229-1237, doi: 10.1212/ WNL.0b013e318214332c, indexed in Pubmed: 21368286.

42. Cobo-Calvo A, Ruiz A, Maillart E, et al. OFSEP and NOMADMUS Study Group. Clinical spectrum and prognostic value of CNS MOG autoimmunity in adults: The MOGADOR study. Neurology. 2018; 90(21): e1858-e1869, doi: 10.1212/WNL.0000000000005560, indexed in Pubmed: 29695592.

43. Akaishi T, Sato D, Nakashima I, et al. MRI and retinal abnormalities in isolated optic neuritis with myelin oligodendrocyte glycoprotein and aquaporin-4 antibodies: a comparative study. J Neurol Neurosurg Psychiatry. 2016; 87(4): 446-448, doi: 10.1136/jnnp-2014-310206.

44. Chen JJ, Flanagan EP, Jitprapaikulsan J, et al. Myelin oligodendrocyte glycoprotein antibody-positive optic neuritis: clinical characteristics, radiologic clues, and outcome. Am J Ophthalmol. 2018; 195: 8-15, doi: 10.1016/j.ajo.2018.07.020, indexed in Pubmed: 30055153.

45. Asseyer S, Hamblin J, Messina S, et al. Prodromal headache in MOG-antibody positive optic neuritis. Mult Scler Relat Disord. 2020; 40: 101965, doi: 10.1016/j.msard.2020.101965, indexed in Pubmed: 32062443.

46. Tajfirouz D, Padungkiatsagul T, Beres $S$, et al. Optic chiasm involvement in AQP-4 antibody-positive NMO and MOG antibody-associated disorder. Mult Scler. 2021: 13524585211011450, doi: 10.1177/13524585211011450, indexed in Pubmed: 33975499.

47. Storoni M, Davagnanam I, Radon M, et al. Distinguishing optic neuritis in neuromyelitis optica spectrum disease from multiple sclerosis: a novel magnetic resonance imaging scoring system. J Neuroophthalmol. 2013; 33(2): 123-127, doi: 10.1097/WNO.0b013e318283c3ed, indexed in Pubmed: 23609766.

48. Ramanathan S, Prelog K, Barnes EH, et al. Radiological differentiation of optic neuritis with myelin oligodendrocyte glycoprotein antibodies, aquaporin-4 antibodies, and multiple sclerosis. Mult Scler. 2016; 22(4): 470-482, doi: $10.1177 / 1352458515593406$, indexed in Pubmed: 26163068.

49. Balloy G, Pelletier J, Suchet L, et al. Société Francophone de la Sclérose en Plaques. Inaugural tumor-like multiple sclerosis: clinical presentation and medium-term outcome in 87 patients. J Neurol. 2018; 265(10): 2251-2259, doi: 10.1007/s00415-018-8984-7, indexed in Pubmed: 30054790.
50. Jolliffe EA, Guo Y, Hardy TA, et al. Clinical and radiologic features, pathology, and treatment of baló concentric sclerosis. Neurology. 2021; 97(4): e414-e422, doi: 10.1212/WNL.0000000000012230, indexed in Pubmed: 34011576.

51. Hardy T, Reddel S, Barnett M, et al. Atypical inflammatory demyelinating syndromes of the CNS. Lancet Neurol. 2016; 15(9): 967-981, doi: 10.1016/s1474-4422(16)30043-6.

52. Lucchinetti CF, Gavrilova RH, Metz I, et al. Clinical and radiographic spectrum of pathologically confirmed tumefactive multiple sclerosis. Brain. 2008; 131(Pt 7): 1759-1775, doi: 10.1093/brain/awn098, indexed in Pubmed: 18535080.

53. Wallner-Blazek M, Rovira A, Fillipp M, et al. Atypical idiopathic inflammatory demyelinating lesions: prognostic implications and relation to multiple sclerosis. J Neurol. 2013; 260(8): 2016-2022, doi: 10.1007/ s00415-013-6918-y, indexed in Pubmed: 23620065.

54. Kalinowska-Lyszczarz A, Tillema JM, Tobin WO, et al. Long-term clinical, MRI, and cognitive follow-up in a large cohort of pathologically confirmed, predominantly tumefactive multiple sclerosis. Mult Scler. 2021 [Epub ahead of print]: 13524585211024162, doi: 10.1177/13524585211024162, indexed in Pubmed: 34212755.

55. Siri A, Carra-Dalliere C, Ayrignac X, et al. Isolated tumefactive demyelinating lesions: diagnosis and long-term evolution of 16 patients in a multicentric study. J Neurol. 2015; 262(7): 1637-1645, doi: 10.1007/s00415-015-7758-8, indexed in Pubmed: 25929666.

56. Saiki S, Ueno Y, Moritani T, et al. Extensive hemispheric lesions with radiological evidence of blood-brain barrier integrity in a patient with neuromyelitis optica. J Neurol Sci. 2009; 284(1-2): 217-219, doi: 10.1016/j.jns.2009.05.022, indexed in Pubmed: 19497587.

57. Zhang YX, Zheng Y, Cai MT, et al. MOG antibody-associated disease presenting with tumefactive lesions and closed-ring enhancement. Acta Neurol Belg. 2021 [Epub ahead of print], doi: 10.1007/s13760021-01695-1, indexed in Pubmed: 34033059.

58. Takai $\mathrm{Y}$, Misu T, Nishiyama $\mathrm{S}$, et al. Hypoxia-like tissue injury and glial response contribute to Balo concentric lesion development. Neurology. 2016; 87(19): 2000-2005, doi: 10.1212/ WNL.0000000000003308, indexed in Pubmed: 27733565.

59. Chien C, Scheel M, Schmitz-Hübsch T, et al. Spinal cord lesions and atrophy in NMOSD with AQP4-IgG and MOG-IgG associated autoimmunity. Mult Scler. 2019; 25(14): 1926-1936, doi: 10.1177/1352458518815596, indexed in Pubmed: 30475082.

60. Mariano R, Messina S, Roca-Fernandez A, et al. Quantitative spinal cord MRI in MOG-antibody disease, neuromyelitis optica and multiple sclerosis. Brain. 2021; 144(1): 198-212, doi: 10.1093/brain/ awaa347, indexed in Pubmed: 33206944.

61. Fadda G, Banwell B, Waters P, et al. Canadian Pediatric Demyelinating Disease Network. Silent new brain MRI lesions in children with mog-antibody associated disease. Ann Neurol. 2021; 89(2): 408-413, doi: 10.1002/ana.25957, indexed in Pubmed: 33210746.

62. Juryńczyk M, Messina S, Palace J. Spinal cord and brain MRI should be routinely performed during follow-up in patients with NMOSD - No. Mult Scler. 2021; 27(1): 15-16, doi: 10.1177/1352458520958599, indexed in Pubmed: 33141633.

63. Kidd D, Barkhof F, McConnell R, et al. Cortical lesions in multiple sclerosis. Brain. 1999; 122(Pt 1): 17-26, doi: 10.1093/brain/122.1.17, indexed in Pubmed: 10050891.

64. Bø L, Vedeler CA, Nyland H, et al. Intracortical multiple sclerosis lesions are not associated with increased lymphocyte infiltration. Mult Scler. 2003; 9(4): 323-331, doi: 10.1191/1352458503ms917oa.

65. Calabrese M, Agosta F, Rinaldi F, et al. Cortical lesions and atrophy associated with cognitive impairment in relapsing-remitting multiple 
sclerosis. Arch Neurol. 2009; 66(9): 1144-1150, doi: 10.1001/archneurol.2009.174, indexed in Pubmed: 19752305.

66. Calabrese M, Poretto V, Favaretto A, et al. Cortical lesion load associates with progression of disability in multiple sclerosis. Brain. 2012; 135(Pt 10): 2952-2961, doi: 10.1093/brain/aws246, indexed in Pubmed: 23065788.

67. Geurts JJG, Roosendaal SD, Calabrese M, et al. MAGNIMS Study Group. Consensus recommendations for MS cortical lesion scoring using double inversion recovery MRI. Neurology. 2011; 76(5): 418-424, doi: 10.1212/WNL.0b013e31820a0cc4, indexed in Pubmed: 21209373.

68. Sethi V, Yousry TA, Muhlert N, et al. Improved detection of cortical MS lesions with phase-sensitive inversion recovery MRI. J Neurol Neurosurg Psychiatry. 2012; 83(9): 877-882, doi: 10.1136/jnnp-2012-303023, indexed in Pubmed: 22807559.

69. Bouman PM, Steenwijk MD, Pouwels PJW, et al. Histopathology-validated recommendations for cortical lesion imaging in multiple sclerosis. Brain. 2020; 143(10): 2988-2997, doi: 10.1093/brain/awaa233, indexed in Pubmed: 32889535.

70. Calabrese M, Oh MiS, Favaretto A, et al. No MRI evidence of cortical lesions in neuromyelitis optica. Neurology. 2012; 79(16): 1671-1676, doi: 10.1212/WNL.0b013e31826e9a96, indexed in Pubmed: 22993282.

71. Takai Y, Misu T, Kaneko K, et al. Japan MOG-antibody Disease Consortium. Myelin oligodendrocyte glycoprotein antibody-associated disease: an immunopathological study. Brain. 2020; 143(5): 1431-1446, doi: 10.1093/brain/awaa102, indexed in Pubmed: 32412053.

72. Höftberger R, Guo Y, Flanagan EP, et al. The pathology of central nervous system inflammatory demyelinating disease accompanying myelin oligodendrocyte glycoprotein autoantibody. Acta Neuropathol. 2020; 139(5): 875892, doi: 10.1007/s00401-020-02132-y, indexed in Pubmed: 32048003.

73. Absinta M, Vuolo L, Rao A, et al. Gadolinium-based MRI characterization of leptomeningeal inflammation in multiple sclerosis. Neurology. 2015; 85(1): 18-28, doi: 10.1212/WNL.0000000000001587, indexed in Pubmed: 25888557.

74. Eisele P, Griebe M, Szabo K, et al. Investigation of leptomeningeal enhancement in MS: a postcontrast FLAIR MRI study. Neurology. 2015; 84(8): 770-775, doi: 10.1212/WNL.0000000000001286, indexed in Pubmed: 25616480.

75. Zurawski J, Tauhid S, Chu R, et al. 7T MRI cerebral leptomeningeal enhancement is common in relapsing-remitting multiple sclerosis and is associated with cortical and thalamic lesions. Mult Scler. 2020; 26(2): 177-187, doi: 10.1177/1352458519885106, indexed in Pubmed: 31714181.

76. Ighani M, Jonas S, Izbudak I, et al. No association between cortical lesions and leptomeningeal enhancement on 7-Tesla MRI in multiple sclerosis. Mult Scler. 2020; 26(2): 165-176, doi: 10.1177/1352458519876037, indexed in Pubmed: 31573837.

77. Asgari N, Flanagan EP, Fujihara $\mathrm{K}$, et al. Disruption of the leptomeningeal blood barrier in neuromyelitis optica spectrum disorder. Neurol Neuroimmunol Neuroinflamm. 2017; 4(4): e343, doi: 10.1212/ NXI.0000000000000343, indexed in Pubmed: 28451627.

78. Kilsdonk ID, Lopez-Soriano A, Kuijer JPA, et al. Morphological features of MS lesions on FLAIR* at $7 \mathrm{~T}$ and their relation to patient characteristics. J Neurol. 2014; 261(7): 1356-1364, doi: 10.1007/s00415-014-7351-6, indexed in Pubmed: 24777693.

79. Maggi P, Absinta M, Grammatico M, et al. Central vein sign differentiates Multiple Sclerosis from central nervous system inflammatory vasculopathies. Ann Neurol. 2018; 83(2): 283-294, doi: 10.1002/ana.25146, indexed in Pubmed: 29328521.

80. Sinnecker T, Dörr J, Pfueller CF, et al. Distinct lesion morphology at 7-T MRI differentiates neuromyelitis optica from multiple sclerosis. Neuro- logy. 2012; 79(7): 708-714, doi: 10.1212/WNL.0b013e3182648bc8, indexed in Pubmed: 22855861.

81. Ciotti JR, Eby NS, Brier MR, et al. Central vein sign and other radiographic features distinguishing myelin oligodendrocyte glycoprotein antibody disease from multiple sclerosis and aquaporin-4 antibody-positive neuromyelitis optica. Mult Scler. 2021 [Epub ahead of print]: 13524585211007086, doi: 10.1177/13524585211007086, indexed in Pubmed: 33870786.

82. Wuerfel J, Sinnecker T, Ringelstein EB, et al. Lesion morphology at 7 Tesla MRI differentiates Susac syndrome from multiple sclerosis. Mult Scler. 2012; 18(11): 1592-1599, doi: 10.1177/1352458512441270, indexed in Pubmed: 22711711.

83. Tallantyre EC, Dixon JE, Donaldson I, et al. Ultra-high-field imaging distinguishes MS lesions from asymptomatic white matter lesions. Neurology. 2011; 76(6): 534-539, doi: 10.1212/WNL.0b013e31820b7630, indexed in Pubmed: 21300968.

84. Mistry N, Abdel-Fahim R, Samaraweera A, et al. Imaging central veins in brain lesions with 3-T T2*-weighted magnetic resonance imaging differentiates multiple sclerosis from microangiopathic brain lesions. Mult Scler. 2016; 22(10): 1289-1296, doi: 10.1177/1352458515616700, indexed in Pubmed: 26658816.

85. Dal-Bianco A, Grabner G, Kronnerwetter C, et al. Slow expansion of multiple sclerosis iron rim lesions: pathology and $7 \mathrm{~T}$ magnetic resonance imaging. Acta Neuropathol. 2017; 133(1): 25-42, doi: 10.1007/ s00401-016-1636-z, indexed in Pubmed: 27796537.

86. Absinta M, Sati P, Masuzzo F, et al. Association of chronic active multiple sclerosis lesions with disability in vivo. JAMA Neurol. 2019; 76(12): 1474-1483, doi: 10.1001/jamaneurol.2019.2399, indexed in Pubmed:31403674.

87. Maggi P, Kuhle J, Schädelin S, et al. Chronic white matter inflammation and serum neurofilament levels in multiple sclerosis. Neurology. 2021; 97(6): e543-e553, doi: 10.1212/WNL.0000000000012326, indexed in Pubmed: 34088875.

88. Tillema JM, Weigand SD, Dayan M, et al. Dark rims: novel sequence enhances diagnostic specificity in multiple sclerosis. AJNR Am J Neuroradiol. 2018; 39(6): 1052-1058, doi: 10.3174/ajnr.A5636, indexed in Pubmed: 29700044.

89. Maggi P, Sati P, Nair G, et al. Paramagnetic rim lesions are specific to multiple sclerosis: an international multicenter $3 T$ MRI study. Ann Neurol. 2020; 88(5): 1034-1042, doi: 10.1002/ana.25877, indexed in Pubmed: 32799417.

90. Sinnecker T, Schumacher S, Mueller K, et al. MRI phase changes in multiple sclerosis vs neuromyelitis optica lesions at 7T. Neurol Neuroimmunol Neuroinflamm. 2016; 3(4): e259, doi: 10.1212/ NXI.0000000000000259, indexed in Pubmed: 27489865.

91. Suthiphosuwan S, Sati $P$, Absinta M, et al. Paramagnetic rim sign in radiologically isolated syndrome. JAMA Neurol. 2020; 77(5): 653-655, doi: 10.1001/jamaneurol.2020.0124, indexed in Pubmed: 32150224.

92. Juryńczyk M, Weinshenker B, Akman-Demir G, et al. Status of diagnostic approaches to AQP4-IgG seronegative NMO and NMO/MS overlap syndromes. J Neurol. 2016; 263(1): 140-149, doi: 10.1007/s00415-0157952-8, indexed in Pubmed: 26530512.

93. Maggi P, Fartaria MJ, Jorge J, et al. CVSnet: A machine learning approach for automated central vein sign assessment in multiple sclerosis. NMR Biomed. 2020; 33(5): e4283, doi: 10.1002/nbm.4283, indexed in Pubmed: 32125737.

94. Barquero G, La Rosa F, Kebiri H, et al. RimNet: a deep 3D multimodal MRI architecture for paramagnetic rim lesion assessment in multiple sclerosis. Neuroimage Clin. 2020; 28: 102412, doi: 10.1016/j. nicl.2020.102412, indexed in Pubmed: 32961401. 